\title{
Relation between seismicity and pre-earthquake electromagnetic emissions in terms of energy, information and entropy content
}

\author{
S. M. Potirakis ${ }^{1}$, G. Minadakis ${ }^{2}$, and K. Eftaxias ${ }^{3}$ \\ ${ }^{1}$ Department of Electronics, Technological Education Institute (TEI) of Piraeus, 250 Thivon \& P. Ralli, 12244, \\ Aigaleo, Athens, Greece \\ ${ }^{2}$ Department of Electronic and Computer Engineering, Brunel Univ. Uxbridge, Middlesex, UB8 3PH, UK \\ ${ }^{3}$ Department of Physics, Section of Solid State Physics, Univ. of Athens, Panepistimiopolis, 15784, Zografos, Athens, Greece
}

Correspondence to: K. Eftaxias (ceftax@ phys.uoa.gr)

Received: 26 December 2011 - Accepted: 16 March 2012 - Published: 25 April 2012

\begin{abstract}
In this paper we show, in terms of Fisher information and approximate entropy, that the two strong impulsive $\mathrm{kHz}$ electromagnetic (EM) bursts recorded prior to the Athens earthquake (EQ) (7 September 1999, magnitude 5.9) present compatibility with the radar interferometry data and the seismic data analysis, which indicates that two fault segments were activated during Athens EQ. The calculated Fisher information and approximate entropy content ratios closely follow the radar interferometry result that the main fault segment was responsible for $80 \%$ of the total energy released, while the secondary fault segment for the remaining $20 \%$. This experimental finding, which appears for the first time in the literature, further enhances the hypothesis for the seismogenic origin of the analyzed $\mathrm{kHz}$ EM bursts.
\end{abstract}

\section{Introduction}

The research related to short-term earthquake (EQ) prediction does not appear to be seen favorably by the scientific community. There have been expressed arguments up to the extreme that it is impossible to have any precursory activity (Uyeda et al., 2009). These views do not appear to be unjustified if one considers the difficulties coupled with the facts that the large EQs are rare, extremely complex phenomena, and there is a great variety of possible pre-seismic patterns.

Although, many issues related to EQ generation are not fully clarified yet, it is reasonably expected that the preparatory process of EQs has various aspects that may be observed before the final event. Therefore, the multi-disciplinary character of the science of EQ prediction is indubitable. In this work, we deal with this subject. Physical fields that are caused by fracture phenomena allow the real-time observation of the evolution of damage of materials under mechanical loading. Crack openings produce electromagnetic (EM) emissions in a wide frequency band from $\mathrm{kHz}$ to $\mathrm{MHz}$. Both laboratory and geophysical-scale experimental data report the detection of these precursors. Monitoring techniques based on these fracture-induced fields are fundamental for the comprehension of fracture mechanism, as well as for the development of models of rock/focal area behavior. EM precursors have not been adequately accepted as valid yet. Two criteria have been recently proposed for the acknowledgment of an observed signal as EQ precursor (Cicerone et al., 2009). The first one was "the reported existence of credible scientific evidence for anomalies in the observables prior to at least some earthquakes" and the other was the existence of "acceptable physical models to explain the existence of the precursor". We will show that the EM precursors under study satisfy the aforementioned two criteria.

We focus on a well-documented pre-seismic EM signal associated with the Athens EQ (7 September 1999, $M=5.9$ ). Two strong impulsive $\mathrm{kHz}$ EM emissions were recorded, with a sampling rate of 1 sample $\mathrm{s}^{-1}$. The anomaly came to an end $9 \mathrm{~h}$ before the main event. The first strong emission lasted for $12 \mathrm{~h} ; 12 \mathrm{~h}$ of background noise were recorded after that, followed by the second strong emission of $17 \mathrm{~h}$ duration (Eftaxias et al., 2001; Papadimitriou et al., 2008). The existence of these $\mathrm{kHz}$ EM signals has been criticized in the past (Pham and Geller, 2002). However, a series of papers that followed, e.g. Eftaxias et al. (2004); Kapiris et al. (2004); Contoyiannis et al. (2005); Karamanos et al. (2006); Papadimitriou et al. (2008); Eftaxias et al. (2008); Potirakis et al. (2012); Minadakis et al. (2012), have further 
supported the view that the detected anomalies were preseismic. It has been repeatedly clarified that such signals have been recorded only in the case of strong surface EQs with epicenters inland (or near the coastline), a fact which provides an explanation for their transmission to the air. Note that the recently proposed fractal geoantenna model (Eftaxias et al., 2004) justifies why there is an increased possibility of detection of such EM radiation, due to the increased radiated power compared to the power radiated in the case of a dipole model.

In terms of seismic energy, as this results from seismic and radar interferometry data, it has been proved that prior to the Athens EQ, two faults were activated. The objective of this paper is to support the hypothesis that the analyzed two $\mathrm{kHz}$ EM anomalies were sourced from the two faults. We show that the information and entropy content included in these two anomalies are quantitatively consistent with the seismic energy released, as it results from radar interferometry (Kontoes et al., 2000), as well as from seismic data analysis (Eftaxias et al., 2001), during the subsequent activation of two faults. The analysis is performed in terms of Fisher information and approximate entropy. We also refer to the already presented (Eftaxias et al., 2001) corresponding analysis for the EM energy for completeness. Finally, we discuss a series of arguments suggesting that the detection of the analyzed $\mathrm{kHz}$ EM activity was part of a series of findings from different disciplines indicating different mechanisms and different phases of the EQ preparation process, further supporting its precursor nature.

\section{Introduction to Fisher information and approximate entropy}

In this section we briefly provide the basic background knowledge and some useful formulae concerning the Fisher information and approximate entropy.

\subsection{Fisher information}

Fisher information provides a powerful tool for the investigation of complex and non-stationary signals (Martin et al., 1999; Potirakis et al., 2012, and references therein). It has been used as a metric of the level of disorder of a system or phenomenon. It behaves inversely to entropy, i.e. increased order is characterized by decreased entropy, but increased Fisher information. It has been employed to study several geophysical and environmental phenomena, divulging its ability to describe the complexity of a system, e.g. Balasco et al. (2008); Telesca et al. (2010), and suggesting its use to identify reliable precursors of critical events (Potirakis et al., 2012, and references therein).

In the case of a discrete measured variable $s_{k}=s\left(t_{k}\right)$, with $t_{k}=k T, k=1,2, \ldots, K$, and $T$ being the sampling period, one can define a set of $N$ disjoint but adjacent intervals (bins) covering the whole range of values between the minimum and maximum observed values of the time series $\left\{s_{k}\right\}$, denoted as $\left\{x_{n}\right\}, n=1,2, \ldots, N$. The corresponding probability for a sample of the time series to belong to the $n$-th interval can be denoted as $p\left(x_{n}\right)$. Then, Fisher information in its discrete form can be expressed (Martin et al., 1999) as

$I_{x}=\sum_{n=1}^{N-1} \frac{\left[p\left(x_{n+1}\right)-p\left(x_{n}\right)\right]^{2}}{p\left(x_{n}\right)}$.

The discrete probability distribution $p\left(x_{n}\right)$ corresponds to the specific values of the unknown underlying probability density function at the center values of the intervals $\left\{x_{n}\right\}$, which are not necessarily of equal length. The probability density function is usually approximated by the histogram, or by the kernel density estimator technique, employing different kernel functions like Gaussian kernel or Epanechnikov kernel (Potirakis et al., 2012, and references therein).

\subsection{Approximate entropy}

Approximate entropy (ApEn) was introduced by Pincus (Pincus, 1991) as a measure of complexity or regularity that is applicable to noisy, medium-sized datasets. Since its introduction, ApEn has been widely applied to a variety of time series of physiological and physical systems and has shown its superiority to most complexity measures such as fractal dimension, Kolmogorov-Sinai entropy, and spectral entropy (Karamanos et al., 2006).

For a time series of a discrete measured variable $s_{k}=s\left(t_{k}\right)$, with $t_{k}=k T, k=1,2, \ldots, N$, and $T$ being the sampling period, we can define $N-m+1$ vectors, each one consisting of $m$ consecutive samples of this time series as

$\boldsymbol{X}_{i}^{m}=\left\{s_{i}, s_{i+1}, \ldots, s_{i+m-1}\right\}, \quad i=1,2, \ldots, N-m+1$.

The main idea is to consider a window of length $m$ running through the time series and forming the corresponding vectors $\boldsymbol{X}_{i}^{m}$. The similarity between the formed vectors is used as a measure of the organization degree of the time series. A quantitative measure of this similarity, $C_{i}^{m}(r)$, is $(N-m+1)^{-1}$ times the number of vectors $\boldsymbol{X}_{j}^{m}$ within a distance $r$ from $\boldsymbol{X}_{i}^{m}$. By calculating it for each $i \leq N-m+1$ and then taking the mean value of the corresponding natural logarithms, $\varphi^{m}(r)$,

$\varphi^{m}(r)=(N-m+1)^{-1} \sum_{i=1}^{N-m+1} \ln C_{i}^{m}(r)$.

ApEn is defined as

$\operatorname{ApEn}(m, r)=\lim _{N \rightarrow \infty}\left[\varphi^{m}(r)-\varphi^{m+1}(r)\right]$.

ApEn is a "regularity statistic" that quantifies the unpredictability of fluctuations in a time series. The presence of repetitive patterns of fluctuation in a time series renders it more predictable than a time series, in which such patterns 

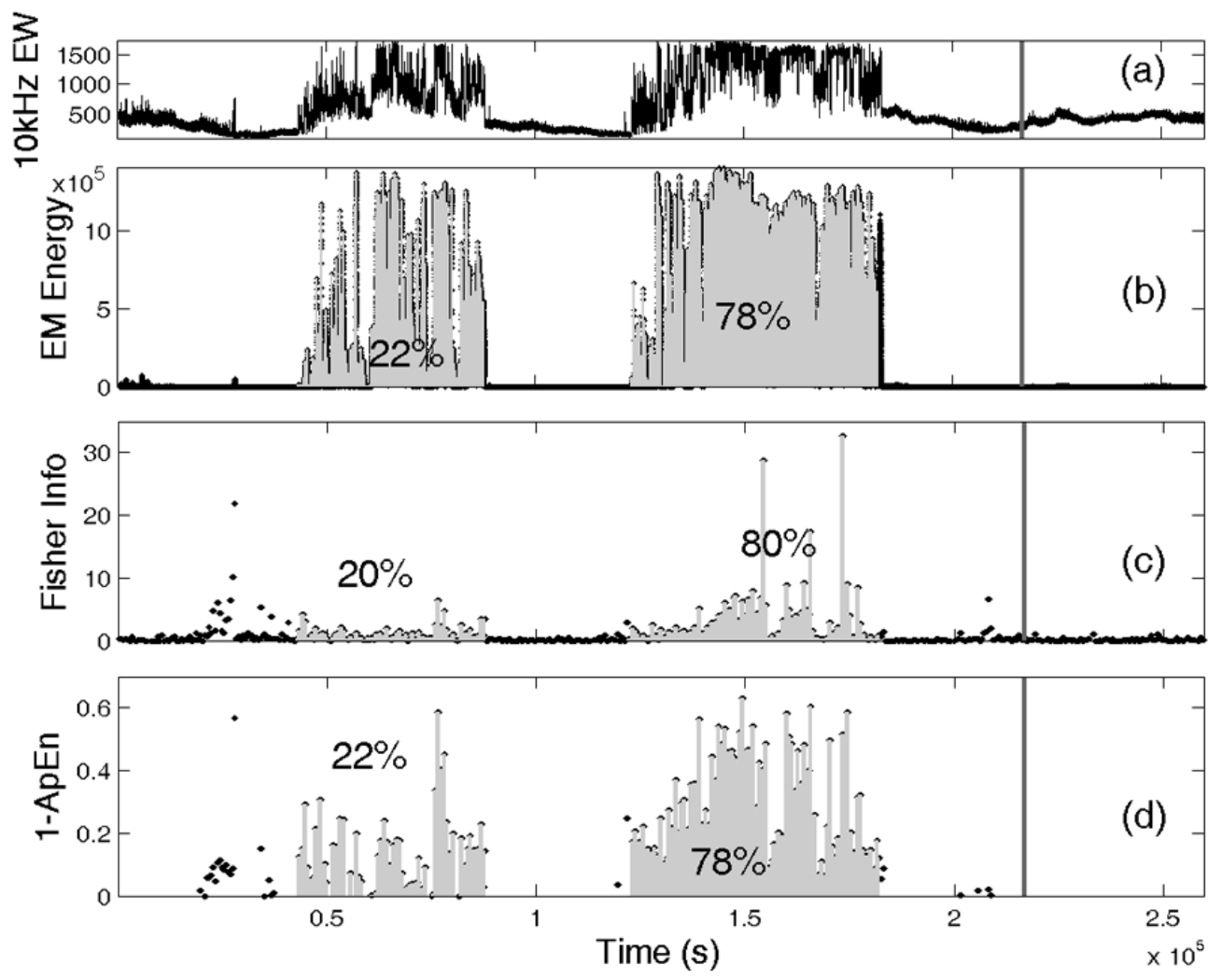

Fig. 1. (a) The two strong impulsive bursts in the tail of the recorded pre-seismic kHz EM emission (10 kHz, East-West, magnetic field strength in arbitrary units) prior to Athens EQ (please refer to Fig. 1 in Papadimitriou et al., 2008). For the specific signal excerpt, the EM Energy (in arbitrary units) (b), the Fisher information (c) and approximate entropy (d) evolution with time is presented. The grey areas indicate the energy, information and 1-ApEn corresponding to the two bursts. The first (left) burst is responsible for the $22 \%$ of the EM energy, the $20 \%$ of the Fisher information, and the $22 \%$ of the ApEn, while the second (right) for the $78 \%$, $80 \%$, and $78 \%$, respectively. All graphs are time-aligned for direct reference. The time of the EQ occurrence is indicated by the thick, vertical grey line.

are absent. A time series containing many repetitive patterns has a relatively small ApEn; a less predictable (i.e. more complex) process has a higher ApEn.

\section{Electromagnetic data analysis}

A way to examine transient phenomena is to analyze the preseismic EM time series into a sequence of distinct time windows. The aim is to discover a clear difference of dynamical characteristics as the catastrophic event is approaching. All employed metrics (Fisher information, approximate entropy and EM energy) were computed here versus time, by dividing the acquired time series in consecutive non-overlapping time windows of 512 samples length and computing all metrics for each one of them. In the case of Fisher information and approximate entropy, the first difference of the raw data time series was analyzed in order to remove the nonstationarities of the first order (Telesca et al., 2011).

The Fisher information (Fig. 1c) and approximate entropy (Fig. 1d), associated with successive time windows, were calculated and their evolution with time was studied. As it can be observed by the outliers of Fig. 1c, the unsmoothed Fisher information time pattern presents quasi-spike-like behavior, due to the signal's abrupt transitions (Telesca et al., 2010). The ApEn was calculated for $m=2$ and $r=0.65 \times$ STD, where STD is the standard deviation of the analyzed time series fragment, allowing fragments with different amplitudes to be compared .

In Fig. 1d, the 1-ApEn is depicted for convenience, considering that the background noise entropy values correspond to entropy equal to 1 . In both Fig. $1 \mathrm{c}$ and d, only the information and entropy values that exceed the level of information and entropy of the background noise are depicted, respectively. This was accomplished by calculating the background noise information and entropy level during a quiet period of the signal, and then "subtracting" them from the calculated Fisher information and ApEn values, respectively.

We observed that there was a significant increase of the Fisher information and a corresponding significant decrease of ApEn values during the two strong EM bursts (the grey areas in Fig. 1c and d, respectively), specifically during the second one. Fisher information and ApEn highlight them efficiently from the normal (background) behavior, indicating their more ordered state (lower complexity). 
If the Fisher information of the $j$-th time window is denoted by $I_{j}$, then the information content of $w$ successive time windows is considered here to be represented by the information sum $I_{\mathrm{s}}=\sum_{j=n}^{n+w} I_{j}, j=n, \ldots, n+w$. In this context, we estimate that Fisher information content of the strong impulsive bursts, in the tail of the pre-seismic EM emission of Fig. 1a, is unevenly distributed between the two bursts. Specifically, the first burst is responsible for $20 \%$ of their total information content, while the second for the remaining $80 \%$ (Fig. 1c). Similarly, the ApEn distribution between the two bursts was calculated to be $22 \%$ vs. $78 \%$ (Fig. 1d).

If the recorded precursory EM time series amplitude is denoted by $A\left(t_{i}\right)$, the amplitude, $A_{\text {fem }}$, of a candidate "fractoelectromagnetic emission" is considered to be the difference $A_{\text {fem }}\left(t_{i}\right)=A\left(t_{i}\right)-A_{\text {noise }}$, where $A_{\text {noise }}$ is the maximum value of the EM recording during a quiet period, namely at a considerable time distance from the time of the EQ occurrence. The corresponding EM energy is estimated by the signal energy, $\varepsilon_{i}=\left(A_{\text {fem }}\left(t_{i}\right)\right)^{2} \times \Delta t$, where $\Delta t$ is the sampling period (here $1 \mathrm{~s}$ ). We considered that a sequence of $k$ successively emerged "fracto-electromagnetic emissions", and $A_{\text {fem }}\left(t_{i}\right), i=m, \ldots, m+k$ represents the EM energy released, $E=\sum_{i=m}^{m+k} \varepsilon_{i}$, during the damage of a structure.

The temporal evolution of the EM energy of the analyzed time series is shown in Fig. 1b. The energy release is also distributed so that the second burst contains $\sim 80 \%$ of the total energy, as already indicated in Eftaxias et al. (2001). To be more precise, the first burst is responsible for the $22 \%$ of their total energy release, while the second for the remaining $78 \%$ (Fig. 1b).

\section{Conclusions and discussion}

We have shown that the ratios of the Fisher information, approximate entropy and EM energy of the two $\mathrm{kHz}$ EM anomalies are consistent with the corresponding seismic energy ratio, as it results from radar interferometry (Kontoes et al., 2000), for the two faults activated during Athens EQ. For the first time a very strong relation between a pre-seismic signal and the ensuing fault activation appears in the literature simultaneously for energy, information and entropy content.

The application of multidisciplinary statistical analysis methods in terms of information and entropy (Papadimitriou et al., 2008; Potirakis et al., 2012) has been sensitively recognized and discriminated the candidate EM precursor under study. However, a stronger indication of the seismogenic origin of the signal would be (a) the presence of several universally holding scaling relations found in fracture and faulting processes, (b) the existence of "acceptable physical models to explain the existence of the precursor" (Cicerone et al., 2009), and (c) the compatibility with precursors from other disciplines. In our case all these three conditions are fulfilled: a. The $\mathrm{kHz}$ EM emissions under study constitute a temporal fractal following the $\mathrm{BBm}$ model with roughness, which is in harmony with the universal spatial roughness of fracture surfaces (Eftaxias et al., 2008). They behave as a "reduced image" of the regional seismicity, and a "magnified image" of laboratory seismicity (Papadimitriou et al., 2008).

b. Both $\mathrm{MHz}$ and $\mathrm{kHz}$ signals are detected prior to large EQs. Moreover, the $\mathrm{MHz}$ radiation precedes the $\mathrm{kHz}$ one, as also observed in the case of the Athens EQ (Kapiris et al., 2004; Contoyiannis et al., 2005). Their generation has been supported by the following "two stage model of EQ generation": The MHz EM emission is thought to be due to the fracture of the highly heterogeneous system that surrounds the family of large high-strength entities distributed along the fault sustaining the system, while the $\mathrm{kHz}$ EM radiation is due to the fracture of the aforementioned large high-strength entities themselves (e.g. Kapiris et al., 2004; Contoyiannis et al., 2005). It has been proposed that the $\mathrm{MHz}$ signal results from a second order phase transition phenomenon, while the $\mathrm{kHz}$ signal results from a nonequilibrium instability phenomenon (Contoyiannis et al., 2005). Moreover, a new perspective to the $\mathrm{kHz}$ generation mechanism has been recently proposed (Minadakis, et al., 2012). In addition, Varotsos et al. (2011) have reported that the occurrence time of a main shock is specified in advance by analyzing in "natural time" the seismicity subsequent to the initiation of the Seismic Electric Signals (SES), which are transient low frequency $(\leq 1 \mathrm{~Hz})$ electric signals that have been repeatedly recorded before earthquakes. This analysis identifies the time when the seismicity approaches the critical state: the main shock was found empirically to follow usually within "a few days up to one week" (Varotsos et al., 2011). It is important to note that the $\mathrm{MHz} / \mathrm{kHz} \mathrm{EM}$ precursors emerged approximately a week up to a few hours before the EQ occurrence. We emphasize that the $\mathrm{MHz}$ EM precursors can also be attributed to a phase transition of second order, as it happens for the seismicity preceding main shocks (Contoyiannis et al., 2005). In the frame of the proposed two stage model, the finally emerged $\mathrm{kHz}$ EM precursors indicate that the occurrence of the prepared EQ is unavoidable. This scheme, namely the appearance of SES following by $\mathrm{MHz}-\mathrm{kHz}$ EM precursory radiations, has been reported before the Athens EQ (Eftaxias et al., 2001).

c. The appearance of signals from other disciplines indicated that an EQ preparation was in process around Athens as well. There was an acceleration of seismicity before the event, closely followed by the corresponding kHz EM emissions (Papadimitriou et al., 2008). Clear TIR (Thermal InfraRed) signals over the area around the 
Athens EQ epicenter were detected from satellites during the last days prior to the Athens EQ (Papadimitriou et al., 2008): after 28 August 1999, a progressive increase (in extension and intensity) of the area affected started, reaching its maximum on 5 September 1999 (i.e. two days before the earthquake) and progressively dissipated after the event. The high-resolution topographic maps for measuring crustal strain accumulated over longer periods of time, obtained by radar interferometry (ERS-2 satellite), predicted the activation of two faults during Athens EQ (Kontoes et al., 2000).

Therefore, the probability of the analyzed signal to be a pre-seismic one seems to be increased. Not only because of the quantitative result that its energy, information and entropy contents are consistent with the seismic energy release during the subsequent activation of two faults, but also because of the presence of pre-seismic activity indications from other disciplines, and the specific sequence of the emerged signals, corresponding to different generation mechanisms and different stages of EQ preparation process (SES, MHz, $\mathrm{kHz})$.

Edited by: M. E. Contadakis

Reviewed by: M. Kachakhidze and another anonymous referee

\section{References}

Balasco, M., Lapenna, V., Lovallo, M., Romano, G., Siniscalchi, A. and Telesca, L.: Fisher information measure analysis of Earth's apparent resistivity, Int. J. Nonlinear Sci., 5, 230-236, 2008.

Cicerone, R. D., Ebel, J. E., and Britton, J.: A systematic compilation of earthquake precursors, Tectonophysics, 476, 371-396, 2009.

Contoyiannis, Y. F., Kapiris, P., and Eftaxias, K.: A Monitoring of a Pre-Seismic Phase from its Electromagnetic Precursors, Phys. Rev. E, 71, 066123, 1-14, 2005.

Eftaxias, K., Kapiris, P., Polygiannakis, J., Bogris, N., Kopanas, J., Antonopoulos, G., Peratzakis, A., and Hadjicontis, V.: Signatures of pending earthquake from electromagnetic anomalies, Geophys. Res. Let., 28, 3321-3324, 2001.

Eftaxias, K., Frangos, P., Kapiris, P., Polygiannakis, J., Kopanas, J., Peratzakis, A., Skountzos, P., and Jaggard, D.: Review and a model of pre-seismic electromagnetic emissions in terms of fractal electrodynamics, Fractals, 12, 243-273, 2004.

Eftaxias, K., Contoyiannis, Y., Balasis, G., Karamanos, K., Kopanas, J., Antonopoulos, G., Koulouras, G., and Nomicos, C.: Evidence of fractional-Brownian-motion-type asperity model for earthquake generation in candidate pre-seismic electromagnetic emissions, Nat. Hazards Earth Syst. Sci., 8, 657-669, doi:10.5194/nhess-8-657-2008, 2008.
Kapiris, P., Eftaxias, K., and Chelidze, T.: Electromagnetic Signature of Prefracture Criticality in Heterogeneous Media, Phys. Rev. Lett., 92, 1-4, 2004.

Karamanos, K., Dakopoulos, D., Aloupis, K., Peratzakis, A., Athanasopoulou, L., Nikolopoulos, S., Kapiris, P., and Eftaxias, K.: Study of pre-seismic electromagnetic signals in terms of complexity, Phys. Rev. E, 74, 1-21, 2006.

Kontoes, C., Elias, P., Sykioti, O., Briole, P., Remy, D., Sachpazi, M., Veis, G., and Kotsis, I.: Displacement field and fault model for the September 7, 1999 Athens earthquake inferred from ERS2 satellite radar interferometry, Geophys. Res. Lett., 27, 3989-3992, 2000.

Martin, M. T., Pennini, F., and Plastino, A.: Fisher's information and the analysis of complex signals, Phys. Lett. A, 256, 173180, 1999.

Minadakis, G., Potirakis, S. M., Nomicos, C., and Eftaxias, K.: Linking electromagnetic precursors with earth- quake dynamics: An approach based on nonextensive frag- ment and self-affine asperity models, Physica A, 391, 2232-2244, doi:10.1016/j.physa.2011.11.049, 2012.

Papadimitriou, K., Kalimeri, M., and Eftaxias, K.: Nonextensivity and universality in the earthquake preparation process, Phys. Rev. E, 77, 1-14, 2008.

Pham, V. N. and Geller, R. J.: Comment on "Signature of pending earthquake from electromagnetic anomalies" by K. Eftaxias et al., Geophys. Res. Lett., 29, 1871, doi:10.1029/2002GL015328, 2002.

Pincus, S. M.: Approximate entropy as a measure of system complexity, Proc. Natl. Acad. of Sci. USA, 88, 2297-2301, 1991.

Potirakis, S. M., Minadakis, G., and Eftaxias, K.: Analysis of electromagnetic pre-seismic emissions using Fisher information and Tsallis entropy, Physica A, 391, 300-306, 2012.

Telesca, L., Lovallo, M., and Carniel, R.: Time-dependent Fisher Information Measure of volcanic tremor before the 5 April 2003 paroxysm at Stromboli volcano, J. Volcan. Geoth. Res., 195, 78$82,2010$.

Telesca, L., Lovallo, M., Hsu, H.-L., and Chen, C.-C.: Analysis of dynamics in magnetotelluric data by using the Fisher-Shannon method, Physica A, 390, 1350-1355, 2011.

Uyeda, S., Nagao, T., and Kamogawa, M.: Short-term EQ prediction: Current status of seismo-electromagnetics, Tectonophysics, 470, 205-213, 2009.

Varotsos, P., Sarlis, N. V., Skordas, E. S., Uyeda, S., and Kamogawa, M.: Natural time analysis of critical phenomena, Proc. Nat. Ac. Sci., 108, 11361-11364, 2011. 